\title{
COSMIC RAY EVENTS AND NATURAL RADIOACTIVITY IN CCD CRYOSTATS
}

\author{
Ralph Florentin-Nielsen, Michael I. Andersen and \\ Niels Bohr Institute for Astronomy, Physics and Geophysics, Copenhagen University \\ Observatory.
}

Sven P. Nielsen

Ris $₫$ National Laboratory, Denmark.

\begin{abstract}
We have found that many materials that are most commonly used in CCD cryostats are weakly radioactive and therefore contribute to what is rather liberally labelled as Cosmic Ray Events. Some standard optical glasses that are extensively used for lenses and optically flat windows such as UBK-7 contain large amounts of potassium 40 , which renders them useless as windows in CCD dewars. Cobalt 60 is sometimes found in excessive amounts in some steel alloys, even in Covar used as a thermal match to silicon. The choice of materials that emit a minimum of ionizing radiation is discussed.
\end{abstract}

\section{INTRODUCTION}

In long CCD exposures one always finds a number of isolated and sharply defined locations on the CCD frame with high exposures - typically a couple of thousand electrons within a few pixels. Such dense spots or tracks are generally labelled Cosmic Ray Events.

In direct imaging these events normally do not pose serious problems, as they can easily be distinguished from stellar images, as they are not circularly symmetric and have considerably sharper profiles than the point spread function, provided that the image scale in the telescope focus is reasonably well sampled by the CCD pixels (2 pixels/FWHM of the PSF or better). If need be Cosmic Ray Events can be removed in the image processing. However, in cases where the tracks coincides spatially with the objects to be studied, they cannot be removed without some loss in signal-to-noise ratio for that object. In spectrographic exposures cosmic ray events are generally a much more serious problem. Exposure times are often quite long, and the spectra and the comparison lines cover a large number of pixels. Hence the risk of having dense tracks right on top of an interesting spectral feature is much higher.

It is therefore important to aim at keeping the rate of Cosmic Ray Events as low as possible. As the majority of Cosmic Rays consist of hard radiation, mainly muons, it is not possible to screen effectively against these at the telescope.

\section{LOCALLY GENERATED RADIATION}

However, not everything that looks like cosmic ray events actually arrive from space. All 
kinds of ionizing radiation can create tracks of similar energy and geometric extent as can cosmic rays. In particular, radioactive materials in the CCD cryostat can be a significant - and sometimes dominant - source of the tracks that are commonly named cosmic ray events.

We have undertaken measurements to identify materials used in dewar construction that contain radioactive isotopes, and also carried out experiments to verify to what extent the remaining tracks are truly of cosmic origin.

Cylindrical samples of $25 \mathrm{~mm}$ diameter of the most commonly used dewar materials were measured for beta-activity using a calibrated Low-level Geiger-Müller counter at the Ris $\$$ National Laboratory, Denmark. The results are shown in Table 1.

\section{TABLE 1}

Beta ray activity for materials commonly used in CCD cryostats.

\begin{tabular}{lll}
\hline \hline Material: & $\begin{array}{l}\text { Concentration } \\
(\mathrm{Bq} / \mathrm{kg})\end{array}$ & $\begin{array}{l}\text { Detection limit (1.65 sigma) } \\
(\mathrm{Bq} / \mathrm{kg})\end{array}$ \\
\hline Copper & 0.00 & 0.11 \\
Covar & 0.03 & 0.07 \\
Brass & 0.06 & 0.07 \\
Bronze & 0.23 & 0.09 \\
Titanium & 0.28 & 0.15 \\
AlMgSi alloy & 1.3 & 0.30 \\
Al alloy used & 1.6 & 0.33 \\
by InfraRed Lab. & & \\
\hline
\end{tabular}

The beta-activity for copper, covar and brass was below the detection limit, whereas the results for the remaining materials lies well above the detection limit. These measurements do not include the identification of the radionuclides in the various samples.

Larger samples were measured in a gamma ray spectrometer, which by analysis of the gamma ray energy spectrum does identify the radionuclides. The results are given in TABLE 2 to one significant digit only since the geometry of the samples did not match exactly that from the calibration.

We have found potassium 40 corresponding to about $4 \mathrm{~g}$ potassium and $0.1 \mathrm{~Bq}$ Radium 226 in the UBK-7 lens (field flattener, originally intended to be the window of the CCD cryostat for the Danish Faint Object Spectrograph and Camera, DFOSC). The Loral $2 \mathrm{k} \times 2 \mathrm{k}$ two side edge buttable CCD, which is mounted in a covar package soldered onto a covar block was found to have a cobalt- 60 contamination of $0.7 \mathrm{~Bq}$. The rest of the results are below the detection limit.

\section{3.. CHALK MINE EXPERIMENT}

To verify that the "cosmic ray events" of our CCD camera were indeed dominated by true 
TABLE 2

Radio isotopes identified by gamma ray spectrometer and their activity in Becquerel (number of disintegrations/sec).

\begin{tabular}{lcccc}
\hline \hline Sample: & $\begin{array}{c}\mathrm{K} \\
(\mathrm{g})\end{array}$ & $\begin{array}{c}\text { Ra-226 } \\
(\mathrm{Bq})\end{array}$ & $\begin{array}{c}\text { Th-232 } \\
(\mathrm{Bq})\end{array}$ & $\begin{array}{c}\text { Co-60 } \\
(\mathrm{Bq})\end{array}$ \\
\hline Steel 37 & $<0.02$ & $<0.05$ & $<0.1$ & $<0.04$ \\
AlCuPb alloy & $<0.02$ & $<0.05$ & $<0.1$ & $<0.04$ \\
Loral 2k CCD & $<0.02$ & $<0.07$ & $<0.1$ & $>0.7$ \\
UBK-7 lens & 4.2 & $>0.1$ & $<0.2$ & $<0.07$ \\
Fused silica & $<0.02$ & $<0.06$ & $<0.1$ & $<0.04$ \\
\hline
\end{tabular}

cosmic radiation and not by any local source we have operated the camera at 37 meters below ground level in the Mönsted chalk mines. This is the deepest mine in Denmark that can be used for the experiment, and although it is not a very impressive depth the attenuation of 37 meters of soil is sufficient to allow conclusive measurements to be carried out. Fig. 1 shows the total rate of cosmic ray events as a function of their energy in ADUs. The conversion factor to electrons is 1.4 electrons/ADU. The solid line shows the energy spectrum for the counts in the laboratory, and the dashed line the counts in the Mönsted mines.

The CCD used was a thick Loral $2 \mathrm{k}$ X $2 \mathrm{k}$ with 15 micron pixels. The count rates are shown in Fig. 1.

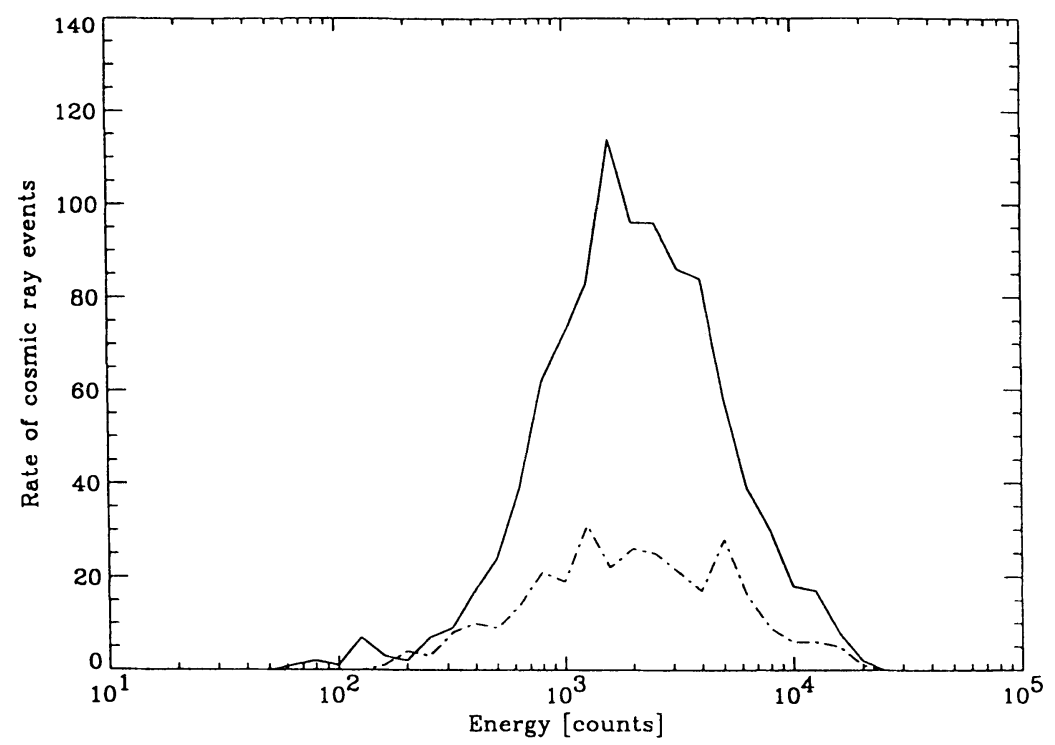

Fig. 1. Energy spectra of Cosmic Ray Events observed at ground level, (sold curve) and $37 \mathrm{~m}$ below ground (dashed curve) 
The count rates were found to be: in the laboratory in Brorfelde: 1.7 events $\mathrm{cm}^{-2} \cdot \mathrm{min}^{-1}$, At 37 meters below ground: 0.56 events ${ }^{\circ} \mathrm{cm}^{-2} \cdot \mathrm{min}^{-1}$.

The spectral distribution is similar in the two sets of measurements with charges ranging from $<100-30,000$ electrons, corresponding to energies of the ionizing radiation of: approximately $300 \mathrm{eV}-100 \mathrm{keV}$, under the assumption that the creation of one electron - hole pair on the average requires $3.5 \mathrm{eV}$ of energy.

Thus, we find a reduction of the count rate by a factor of 3.0. We can therefore safely state that counts due to radioactive contamination is less than one third of what we observe at ground level, and the fact that the energy spectrum is apparently unaltered led us to assume the radioactive counts are well below one third of the count rate found at ground level. For comparison a UBK-7 lens was mounted instead of the fused silica window yielding a count rate of 7.0 events $\mathrm{cm}^{-}{ }^{2} \cdot \mathrm{min}^{-1}$ which is more than 12 times higher than the upper limit to the radioactive count rate with the fused silica window in place.

\section{CONCLUSIONS}

Aluminum, which is the most commonly used material in dewar design has been found to be radioactive to a rather varying degree. First of all, aluminum does contain small traces of uranium 238, but the amount of uranium depends strongly upon where it was mined. A large proportion of all aluminum sold today contains recycled aluminum that has been exposed to radiation from post World War 2 atomic bomb detonations and contain radium. One precaution when using aluminium would be to specify virgin aluminum and to test the material before use.

Electrolytic copper, magnesium and fiber glass composits are ideal materials for use close to the CCD detector from a radiation point of view. Magnesium is highly flammable and chemically very active and is therefore not suited for cryostats. Fiber glass lacks the electrical conductivity which is needed to provide a Faraday cage around the CCD. This leaves copper as the preferred material for the parts of the cryostat that are close to the detector, i.e. the camera head, (ref. Fig. 2). The thermal radiation shield is made of gold plated copper. We shall try to electrolytically deposit a layer of copper on the inside of the camera head made of aluminum.

Covar is a steel alloy with a good match to the thermal expansion coefficient of silicon. We have found large differences in the samples with respect to contents of radioactive cobalt. The new packages for our three side buttable Loral $2 \mathrm{k}$ x $2 \mathrm{k}$ CCDs will be made of "Invar36", which is similar to covar, but has been found to have an even better match to the thermal expansion of silicon at temperatures around $-100^{\circ} \mathrm{C}$. To verify whether the invar 36 at hand is significantly radioactive we intend to machine a sample of the material to the same dimensions as the cryostat window and insert it instead of the fused silica window and compare the count rates in much the same way as we did with the UBK-7 lens. This way, by bringing up the sample close in front of the CCD itself, one can always effectively test the material for any contamination that would affect the observed rate of cosmic ray events without having to utilize beta counters or gamma spectrometers. 


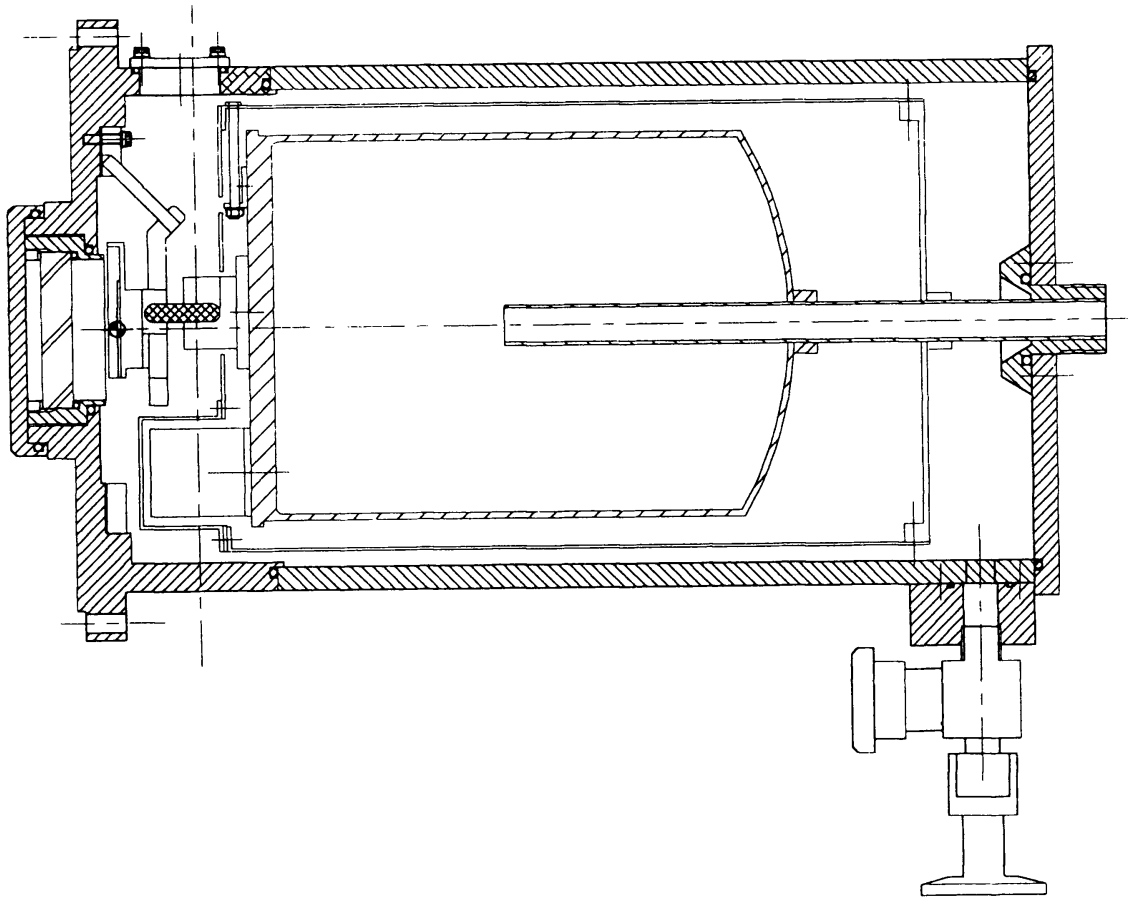

Fig. 2. Liquid Nitrogen cooled Cryostat for Loral and Tektronix CCDs.

\section{DISCUSSION}

YOUNG: It seems you are seeing problems we already had 30 years ago with magnetic shields for PMTs. The steel manufacturers put Co60 in their furnace linings; when the radioactivity goes away, it is time to replace the lining. Of course the Co60 ends up in the steel.

FLORENTIN-NIELSEN: Yes, this is the same problem. However, the CCD detector is more sensitive to a variety of radio isotopes. 